OPEN ACCESS

Edited by:

Fanxiu Zhu,

Florida State University, United States

Reviewed by:

Wenwei Li,

Yale University, United States

Yoshihiro Izumiya,

University of California, Davis,

United States

*Correspondence:

Myung-Shin Lee

imslee@g.eulji.ac.kr

Specialty section:

This article was submitted to

Virology,

a section of the journa

Frontiers in Microbiology

Received: 17 September 2021

Accepted: 19 November 2021

Published: 16 December 2021

Citation:

Kang S-K, Lee M-J, Ryu H-H,

Lee $J$ and Lee M-S (2021) Dimethyl

Sulfoxide Enhances Kaposi's

Sarcoma-Associated Herpesvirus

Production During Lytic Replication.

Front. Microbiol. 12:778525.

do: $10.3389 /$ fmicb.2021.778525

\section{Dimethyl Sulfoxide Enhances Kaposi's Sarcoma-Associated Herpesvirus Production During Lytic Replication}

\author{
Su-Kyung Kang', Myung-Ju Lee ${ }^{1}$, Ho-Hyun Ryu' ${ }^{1}$, Jisu Lee ${ }^{1}$ and Myung-Shin Lee ${ }^{1,2 *}$ \\ ${ }^{1}$ Department of Microbiology and Immunology, Eulji University School of Medicine, Daejeon, South Korea, ${ }^{2}$ Eulji Biomedical \\ Science Research Institute, Eulji University School of Medicine, Daejeon, South Korea
}

Kaposi's sarcoma-associated herpesvirus (KSHV) is an etiologic agent of Kaposi's sarcoma, primary effusion lymphoma, and multicentric Castleman disease. In studies of $\mathrm{KSHV}$, efficient virus production and isolation are essential. Reactivation of KSHV can be initiated by treating latently infected cells with chemicals, such as 12-O-tetradecanoylphorbol-13-acetate and sodium butyrate. These chemicals have been used as tools to induce lytic replication and viral production in KSHV-producing cell lines. Dimethyl sulfoxide (DMSO) is an organosulfur compound that is frequently used as an aprotic solvent similar to water. In experiments exploring signaling pathways in KSHV-infected cells, DMSO treatment alone as a vehicle affected the lytic gene expression of KSHV. However, to the best of our knowledge, the effects of DMSO on KSHV-producing cells have not yet been reported. Therefore, in this study, we investigated whether DMSO could be used as a reagent to enhance viral production during lytic replication in KSHVproducing cells and assessed the underlying mechanisms. The effects of DMSO on KSHV production were analyzed in iSLK BAC16 cells, which have been widely used for recombinant KSHV production. We found that the production of KSHV virions was significantly increased by treatment with DMSO during the induction of lytic replication. Mechanistically, lytic genes of KSHV were enhanced by DMSO treatment, which was correlated with virion production. Additionally, DMSO induced the phosphorylation of JNK during Iytic replication, and inhibition of JNK abolished the effects of DMSO on Iytic replication and virion production. Our findings showed that additional treatment with DMSO during the induction of Iytic replication significantly improved the yield of KSHV production.

Keywords: KSHV, DMSO, herpesvirus, lytic replication, viral production

\section{INTRODUCTION}

Kaposi's sarcoma-associated herpesvirus (KSHV) is a gammaherpesvirus belonging to the family Herpesviridae (Chang et al., 1994). Kaposi's sarcoma (Boshoff and Weiss, 2002), multicentric Castleman disease (Powles et al., 2009), primary effusion lymphoma (Cesarman et al., 1995), and inflammatory cytokine syndrome are known to be associated with KSHV (Polizzotto et al., 2016). 
The isolation of virions is a necessary process for studying KSHV. Similar to other herpesviruses, the life cycle of KSHV consists of two phases: latent and lytic replication cycles (Yan et al., 2019). Virions are produced only during lytic replication. Therefore, efforts have been made to extract large amounts of virus by effectively inducing lytic replication from KSHVharboring cells, and various chemicals have been tested. 12O-Tetradecanoyl-phorbol-13-acetate induces lytic replication of KSHV by activating the mitogen-activated protein kinase (MAPK)/extracellular signal-regulated kinase (ERK) pathway (Cohen et al., 2006). Sodium butyrate activates the KSHV ORF50 promoter by inducing histone $\mathrm{H} 3$ and $\mathrm{H} 4$ hyperacetylation (Lu et al., 2003). Additionally, inomycin (Chang et al., 2000), epinephrine/norepinephrine (Chang et al., 2005), and valproic acid are potent inducers of KSHV reactivation (Shin et al., 2014).

Dimethyl sulfoxide (DMSO) is a polar aprotic molecule with a strong affinity for water. DMSO exhibits a rapid penetration effect and enhances the penetration of other substances. Moreover, the low systemic toxicity of DMSO enables its wide application as a solvent (Brayton, 1986). DMSO has been shown to have various effects on human cellular processes and the epigenetic landscape, such as DNA methylation, mRNA and protein expression, and microRNA expression (Verheijen et al., 2019). In several enveloped viruses, such as influenza A virus, Newcastle disease virus, and Semliki Forest virus, DMSO has a stimulating effect on virus yield (Scholtissek and Muller, 1988). Furthermore, previous studies have demonstrated that DMSO can affect the human herpesvirus life cycle. In herpes simplex virus-1, DMSO blocks productive infection by inhibiting viral DNA replication and viral mRNA expression (Aguilar et al., 2002). In human cytomegalovirus, DMSO enhances virion production in virusinfected cells (Tanaka et al., 1985; West et al., 1988). However, no studies have investigated the effects of DMSO on KSHV.

In our in vitro experiments with KSHV, we found that KSHVharboring cells treated with DMSO as a control vehicle for chemicals showed alterations in lytic gene expression. Therefore, we wondered whether DMSO may have positive effects on $\mathrm{KSHV}$ production in KSHV-producing cells. Accordingly, in this study, we investigated whether DMSO could be used as an enhancing agent for the lytic replication and production of KSHV. Our results revealed that DMSO enhanced KSHV production in iSLK BAC16 cells during induced lytic replication through increased phosphorylation of c-Jun N-terminal kinase (JNK), suggesting that DMSO could be an effective enhancing agent for KHSV production.

\section{MATERIALS AND METHODS}

\section{Cell Culture and Reagents}

iSLK BAC16 cells were cultured in Dulbecco's Modified Eagle's Medium (DMEM)/high glucose (Welgene, Gyeongsan, South Korea) with $10 \%$ fetal bovine serum (FBS; GenDEPOT, Katy, TX, United States) and $1 \%$ antibiotic-antimycotic (Invitrogen, Waltham, MA, United States). Hygromycin B (1.2 $\mathrm{mg} / \mathrm{mL}$; Invitrogen), geneticin $(250 \mu \mathrm{g} / \mathrm{mL}$; Invitrogen), and puromycin $(1 \mu \mathrm{g} / \mathrm{mL}$; Invitrogen) were added and cultured to maintain the latent infection of iSLK BAC16. Human umbilical vein endothelial cells (HUVECs) were purchased from PromoCell (Heidelberg, Germany) and cultured in endothelial cell growth medium 2 (PromoCell) containing cell growth supplements. BCBL-1 cells were cultured in RPMI 1640 (Biowest, Riverside, MO, United States) supplemented with 10\% FBS. All cells were cultured at $37^{\circ} \mathrm{C}$ in a humidified atmosphere containing 95\% air and 5\% $\mathrm{CO}_{2}$. SP600125 was purchased from Calbiochem (San Diego, CA, United States). DMSO was purchased from LPS Solution (Daejeon, South Korea).

\section{Virus Isolation and Infection}

iSLK BAC16 cells harboring recombinant KSHV BAC16 were used to produce virions (Brulois et al., 2012). iSLK BAC16 cells were treated with $1.2 \mathrm{mM}$ sodium butyrate (Sigma, Burlington, MA, United States) and $50 \mu \mathrm{g} / \mathrm{mL}$ doxycycline (Sigma) for $48 \mathrm{~h}$ to induce lytic replication. Upon induction of lytic replication, DMSO was added into culture media together with sodium butyrate and doxycycline at $0.1 \%, 0.5 \%$, or $1 \%$ of the total volume. For virus isolation, the culture medium was collected and centrifuged at $300 \times \mathrm{g}$ for $10 \mathrm{~min}$ at $4^{\circ} \mathrm{C}$ to remove cell debris from the culture supernatant. The supernatant was again centrifuged at $2,000 \times g$ for $10 \mathrm{~min}$ at $4^{\circ} \mathrm{C}$, and the supernatant was collected. The supernatant was collected and centrifuged at $100,000 \times g$ for $1 \mathrm{~h}$ at $4^{\circ} \mathrm{C}$. The virus pellet was resuspended in cold phosphate-buffered saline (PBS) and stored at $-80^{\circ} \mathrm{C}$ until the virus stock was used. KSHV infection was performed as described previously (Kang et al., 2021). Briefly, the prepared KSHV stock was added to Gibco Opti-MEM (Invitrogen) containing $5 \mu \mathrm{g} / \mathrm{mL}$ polybrene (Santa Cruz Biotechnology, Santa Cruz, CA, United States). HUVECs were seeded in 6-well culture plates the day before KSHV infection. KSHV infection was performed by centrifugation at $2,600 \mathrm{rpm}$ for $1 \mathrm{~h}$ at $25^{\circ} \mathrm{C}$. After centrifugation, the medium was changed to endothelial cell growth medium 2 (PromoCell), and HUVECs were then incubated overnight at $37^{\circ} \mathrm{C}$ in a humidified atmosphere containing $5 \% \mathrm{CO}_{2}$.

\section{Flow Cytometry Analysis of Kaposi's Sarcoma-Associated Herpesvirus Infectivity}

Human umbilical vein endothelial cells infected with KSHV BAC16 were detached from culture wells with trypsinethylenediaminetetraacetic acid (EDTA) solution (Invitrogen) and neutralized using a culture medium. After washing with $1 \times$ PBS, the cells were resuspended in PBS containing $1 \%$ FBS. Because cells infected with the recombinant KSHV BAC16 expressed green fluorescence protein (GFP), infected cells were analyzed using a Guava easyCyte flow cytometer (Luminex Corporation, Austin, TX, United States) and InCyte 3.1 software (Luminex Corporation).

\section{Western Blotting}

Lysis buffer $(20 \mathrm{mM}$ Tris- $\mathrm{HCl}, 150 \mathrm{mM} \mathrm{NaCl}, 10 \mathrm{mM}$ EDTA, $1 \mathrm{mM}$ EGTA, and $1 \%$ Triton X-100) was prepared for cell lysates. To inhibit endogenous and exogenous protease and 
phosphatase inhibitors, $0.1 \mathrm{mM}$ sodium fluoride, $0.1 \mathrm{mM}$ phenylmethylsulfonyl fluoride, $1 \mathrm{mM}$ beta-glycerophosphate, and $0.1 \mathrm{mM}$ sodium orthovanadate were added to the lysis buffer immediately before cell lysis. The cell lysate was incubated on ice for $30 \mathrm{~min}$ and then centrifuged at 13,000 rpm for $10 \mathrm{~min}$ at $4^{\circ} \mathrm{C}$. The supernatants were collected, and the protein concentrations were measured using a bicinchoninic acid assay (Pierce, Rockford, IL, United States). Proteins were separated by sodium dodecyl sulfate polyacrylamide gel electrophoresis on $10 \%$ or $12 \%$ gels and transferred to nitrocellulose membranes. The membranes were blocked overnight at $4^{\circ} \mathrm{C}$ with TBST ( $1 \times$ Tris-buffered saline containing $0.1 \%$ Tween 20$)$ containing $5 \%$ skim milk. The primary antibody was diluted in $5 \%$ bovine serum albumin/TBST and reacted with the sample by rocking at $4^{\circ} \mathrm{C}$ overnight. The secondary antibody diluted with TBST containing $5 \%$ skim milk was reacted on a rocker for $1 \mathrm{~h}$ at room temperature. Immunolabeled proteins were detected using Clarity Western ECL Substrate (Bio-Rad Laboratories, Hercules, CA, United States) and an Amersham ImageQuant 800 biomolecular imager (GE Healthcare, Chicago, IL, United States). Immunodetection was performed using rabbit antibodies against HHV8 ORF50 (Bioss, Woburn, MA, United States, bs-0860R), total AKT (Bioss, bs-5050R), total p38 MAPK (Bioss, bs-50503R), phosphorylated p38 MAPK (Thr180/Tyr182, Bioss, bs-50486R), or phosphorylated ERK (Thr202/Tyr204, Bioss, bs-50534R); mouse antibodies against HHV-8 K8.1A/B (sc-65446), JNK (total JNK, sc-7345) or phosphorylated JNK (Thr183/Tyr185, sc-6254, all from Santa Cruz Biotechnology); mouse antibodies against $\beta$-actin (Sigma, A-1978) or KSHV ORF45 (Invitrogen, MA514769); mouse antibodies against KSHV ORF65 (a gift from Dr. Shou-Jiang Gao); and rat antibodies against HHV8 LANA (Abcam, Cambridge, United Kingdom, ab4103). Horseradish peroxidase (HRP)-conjugated goat anti-rabbit IgG (Bethyl, Montgomery, TX, United States, A120-101P), HRP-conjugated goat anti-mouse IgG (Bethyl, A90-101P), and HRP-conjugated goat anti-mouse IgG (Bethyl, A110-105P) were used as secondary antibodies. Protein bands were quantified by western blot analysis using Image Lab 6.0 software (Bio-Rad Laboratories).

\section{RNA Isolation, cDNA Synthesis, and Reverse Transcription-Quantitative Polymerase Chain Reaction Analysis}

Total RNA was isolated from cultured cells using a Ribospin II RNA Isolation Kit (GeneAll Biotechnology, Seoul, South Korea). cDNA was synthesized using PrimeScript RT Master Mix (Takara, Shiga, Japan) at $37^{\circ} \mathrm{C}$ for $10 \mathrm{~min}$. qPCR was performed using Takara TB Green FAST qPCR Mix (Takara). Cycling conditions were as follows: $95^{\circ} \mathrm{C}$ for $30 \mathrm{~s}$, amplification for 40 cycles $\left(95^{\circ} \mathrm{C}\right.$ for $5 \mathrm{~s}$ and $60^{\circ} \mathrm{C}$ for $\left.30 \mathrm{~s}\right)$, and melting curve analysis to confirm the specificity of the amplified product. All samples were run in triplicate, and gene expression levels were normalized to glyceraldehyde 3-phosphate dehydrogenase (GAPDH) expression. qPCR was performed and analyzed using a CFX96 real-time system (Bio-Rad Laboratories) and Bio-Rad CFX Manager software version 3.1 (Bio-Rad Laboratories). Data analysis was carried out as described previously (Yoo et al., 2005).
qPCR was performed using the following primers: human GAPDH, sense (5'-GGTATCGTGGAAGGACTC- $\left.3^{\prime}\right)$ and antisense $\quad\left(5^{\prime}\right.$-GTAGAGGCAGGGATGATG- $\left.3^{\prime}\right)$; KSHV LANA, sense (5'-TTGTGTATATGTGTATTGTCAGAA-3') and antisense (5'-AACTTAACTATGGAAGATTGTAGG-3'); KSHV vCyclin, sense (5'-GCCTCACGCCTATTTCTA-3') and antisense (5'-TTCTCCTGGTCTATAAGTTCTT- $\left.3^{\prime}\right)$; KSHV ORF50, sense (5'-CGCTGTTGTCCAGTATTC-3') and antisense (5'-AGAAGGTGACGGTATATCC-3'); KSHV K8, sense (5'-GCCTGCGTCTGTAGTTAA-3') and antisense (5'-TCCTTATGTGCCTCCAATC-3'); KSHV ORF59, sense $\left(5^{\prime}\right.$-TTAGCCTGGAGTCCTTAATC- $\left.3^{\prime}\right)$ and antisense (5'-GCA CACCTTCCACTTCTA-3'); KSHV ORF74, sense (5'-GCGATA GATATACTGCTCCT- $\left.3^{\prime}\right)$ and antisense (5' -CATCAACACTTCT GCCAAT- $\left.3^{\prime}\right)$; KSHV K8.1, sense (5'-TAAACCCACAGCCC ATAG- $\left.3^{\prime}\right)$ and antisense (5'-CCACCACTACAACGACTA- $\left.3^{\prime}\right)$; KSHV ORF65, sense (5'-ACTATCTCGTGTTCTTAATTGC-3') and antisense (5'-ATGATCCCGCCTTTGAAT- $\left.3^{\prime}\right)$. All primers were synthesized by Genotech (Daejeon, South Korea).

\section{Analysis of Kaposi's Sarcoma-Associated Herpesvirus Genome Copy Number}

For the relative quantification of intracellular KSHV genome DNA, KSHV-infected cells were lysed at $4 \mathrm{~h}$ of postinfection using a DNeasy Blood and Tissue kit (Qiagen, Hilden, Germany) according to the manufacturer's recommendations. To analyze the virion copy number of the isolated $\mathrm{KSHV}$, the supernatants of iSLK BAC16 cells with induction of lytic replication were collected and centrifuged at $100,000 \times g$ for $1 \mathrm{~h}$. The pellet was resuspended in 1X DNase buffer and then treated by RQ1 RNase-free DNase I (Promega, Madison, WI, United States) at $37^{\circ} \mathrm{C}$ for $1 \mathrm{~h}$. DNA was extracted using the DNeasy Blood and Tissue kit (Qiagen). PCR analysis was carried out using the Takara TB Green FAST qPCR Mix (Takara, Shiga, Japan) with primers ORF26F (5' GAC TCT TCG CTG ATG AAC TGG $3^{\prime}$ ) and ORF26R (5' AGC ACT CGC AGG GCA GTA CG 3') targeting KSHV ORF26 (Genotech, Daejun, South Korea). qPCR reaction conditions and data analysis followed the RT-qPCR as mentioned above. The number of viral DNA molecules was calculated from a standard curve constructed from serial dilutions of a known amount of pUC19 vector containing KSHV ORF26.

\section{Immunofluorescence Assay}

Immunofluorescence assay was performed as described previously with minor modifications (Kang et al., 2021). Mouse antibodies against HHV-8 K8.1A/B (Santa Cruz Biotechnology, sc-65446) and KSHV ORF45 (Invitrogen, MA5-14769) were used as primary antibodies.

\section{Lactate Dehydrogenase Assay}

Cytotoxicity was analyzed by a Cytotoxicity Detection kit PLUS (LDH, Roche, Basel, Switzerland) according to the manufacturer's protocol. Cells were seeded at 3,000 cells/100 $\mu \mathrm{l}$ in a 96 well plate. The next day, SP600125 was treated with the indicated concentrations. After $24 \mathrm{~h}$, the supernatant was transferred into 
the corresponding wells of a 96-well plate. The cultured cells with each condition were treated with the enclosed lysis solution for $15 \mathrm{~min}$ as a positive control. The dye solution-catalyst mixture was treated and incubated at room temperature for $10 \mathrm{~min}$. The reaction was terminated with a stop solution, and absorbance was measured at $490 \mathrm{~nm}$.

\section{Knockdown of C-Jun N-Terminal Kinase by Small Interfering RNA Transfection}

Transfection of small interfering RNA (siRNA) was performed using Lipofectamine TM $^{\text {RNAiMAX Transfection Reagent }}$ (Thermo Fisher Scientific) according to the manufacturer's instructions. Briefly, cells were seeded in 6-well plates with DMEM containing 10\% FBS. The next day, the transfection reagent was diluted using Opti-MEM (Thermo Fisher Scientific). siRNA was treated at $100 \mu \mathrm{M}$. After $24 \mathrm{~h}$ post-transfection, cells were induced using sodium butyrate and Doxycycline. After $24 \mathrm{~h}$ post-transfection, cells were induced using sodium butyrate and Doxycycline. Predesigned siRNAs for JNK (MAPK8, 5599) and scrambled siRNA (AccuTarget negative control siRNA) were purchased from Bioneer Corporation (Daejeon, South Korea).

\section{Statistical Analysis}

Results are shown as means \pm standard deviations. The twotailed Student's $t$ test was used to assess the significance of difference between groups. Statistical significance at $P$ values of $<0.05$ and $<0.01$ is indicated by ${ }^{*}$ and ${ }^{* *}$, respectively.

\section{RESULTS}

\section{Treatment With Dimethyl Sulfoxide During Lytic Replication Increased the Infectivity of Kaposi's Sarcoma-Associated Herpesvirus Extracted From iSLK BAC16 Cells Through the Enhanced Production of \\ Virions}

To investigate the effects of DMSO on KSHV-producing iSLK BAC16 cells, we extracted KSHV from iSLK BAC16 cells treated with various concentrations of DMSO during lytic replication and analyzed their infectivity in HUVECs. The schematic processes of virus production and analysis are presented in Figure 1. After treatment of DMSO to iSLK BAC16 cells, cell viability was analyzed (Figure 1). While DMSO alone did not affect cell viability, DMSO with inducing agents for lytic replication showed a significant decreased the cell viability with dose-dependent manners. HUVECs were infected with the same volume of extracted KSHV, and their infectivity was analyzed

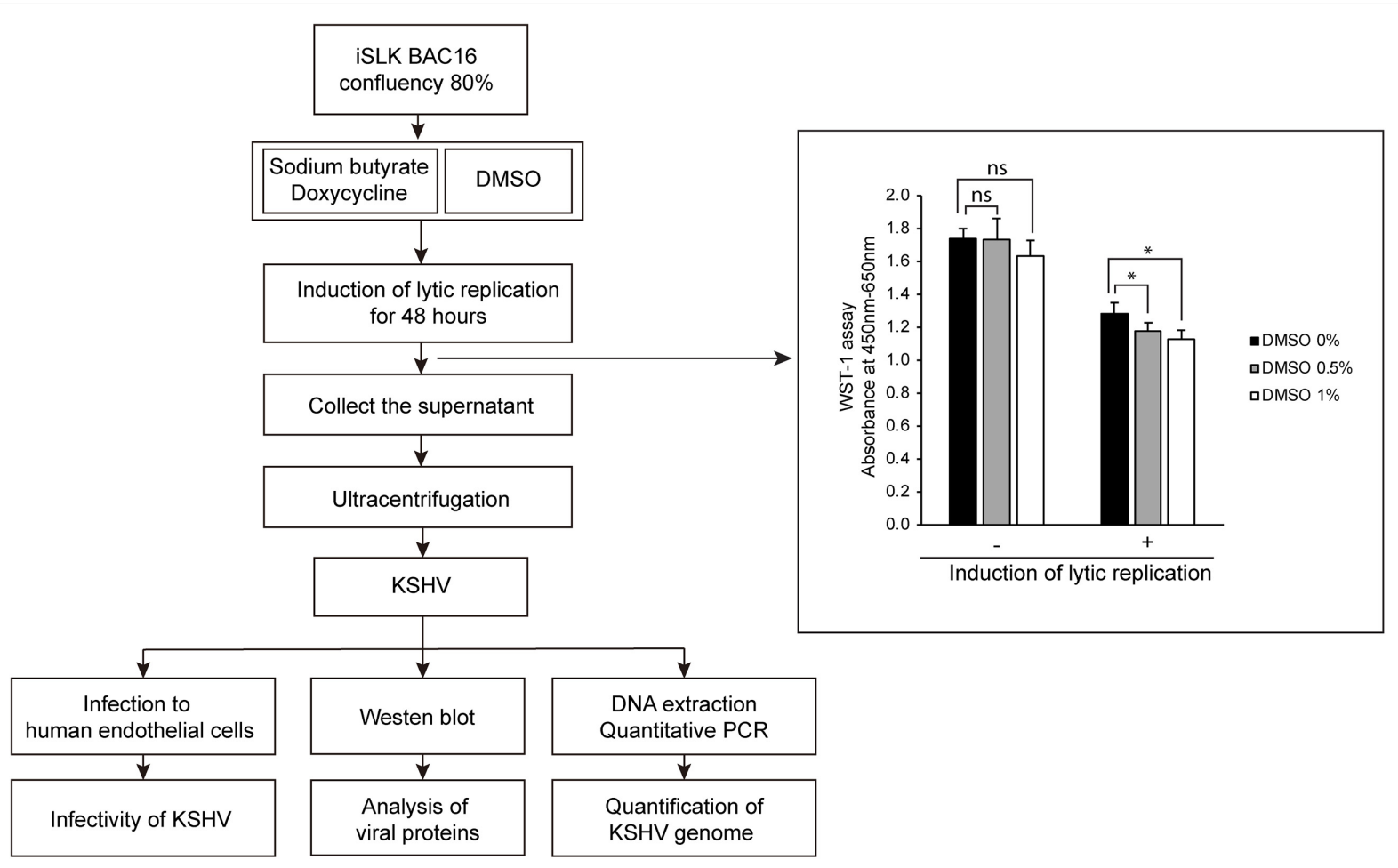

FIGURE 1 | Schematic diagram of the experimental processes and cell viability of iSLK BAC16 cells by treatment of DMSO. Lytic replication was induced by sodium butyrate and doxycycline for $48 \mathrm{~h}$. DMSO was added into culture media together with sodium butyrate and doxycycline at various concentrations. During lytic replication, cells were treated with various concentrations of DMSO to investigate its effects on virus production. After DMSO treatment, cell viabilities were analyzed by WST-1 assay. Data are shown as the mean $\pm \mathrm{SD}, n=3$, ns, not significant; ${ }^{*} p<0.05$. The produced KSHV was extracted by differential centrifugation and analyzed by infection of HUVECs, western blotting, and quantitative PCR (qPCR). 
A

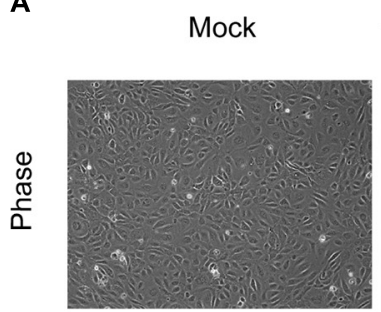

品

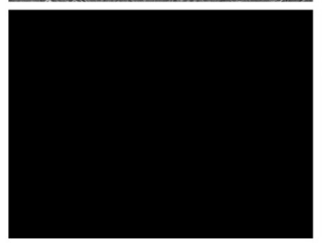

KSHV produced with DMSO
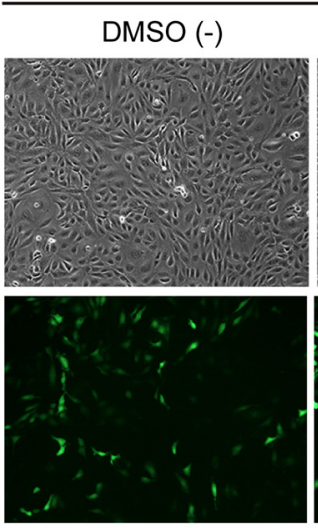

DMSO $0.1 \%$
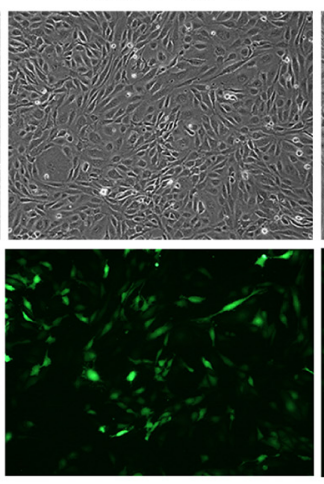

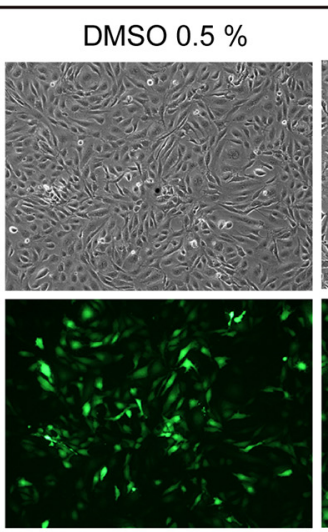

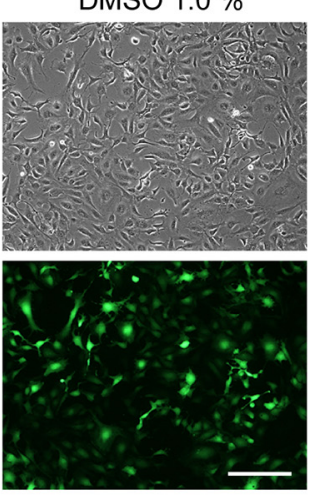

B

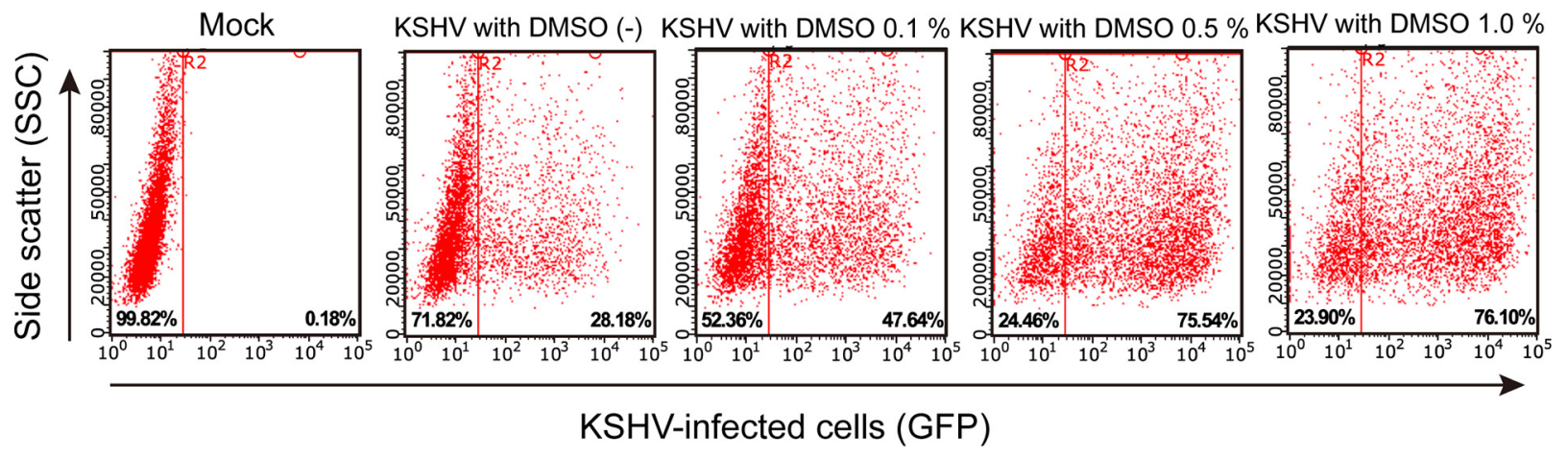

C

D

E
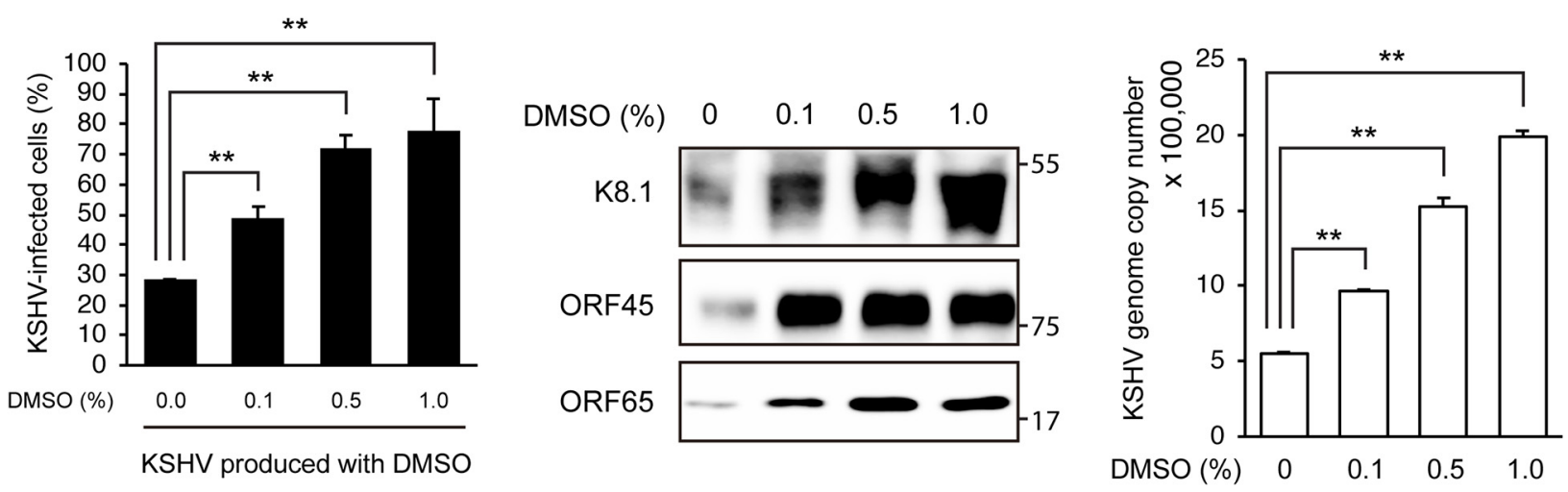

FIGURE 2 | DMSO enhanced KSHV production in iSLK BAC16 cells. KSHV was harvested from iSLK BAC16 cells treated with various concentrations of DMSO during lytic replication. The same volume of KSHV from each group was used to infect HUVECs. (A) GFP expression of KSHV-infected cells in fluorescence microscopy. After $24 \mathrm{~h}$ of infection, infectivity was measured by analysis of GFP expression using fluorescence microscopy. Scale bar $=250 \mu \mathrm{m}$. (B) Representative results of the flow cytometric analysis of GFP expression in KSHV-infected HUVECs. KSHV-infected cells from panel (A) were detached and analyzed by flow cytometry. $X$ - and $Y$-axis indicate GFP expression and side scatter, respectively. The numbers on the right lower corners of each panel indicate the percentage of GFP-positive cells. (C) The flow cytometric analysis of GFP expression in KSHV-infected HUVECs. Data are shown as the mean \pm SD, $n=3,{ }^{\star *} p<0.01$. (D) Western blot analysis of KSHV proteins of the isolated KSHV from iSLK BAC16 cells. After induction of lytic replication, KSHV was extracted from the supernatants of iSLK BAC16 cells. iSLK BAC16 cells were treated with the indicated concentrations of DMSO during lytic replication. The same volume of extracted KSHV from each experimental condition was analyzed. (E) Analysis of KSHV genome copy numbers from the produced virions. Genomic DNA was isolated from the extracted virions and analyzed by GPCR with specific primers targeting KSHV ORF26. Data are shown as the mean $\pm \mathrm{SD}, n=3,{ }^{* *} p<0.01$. 

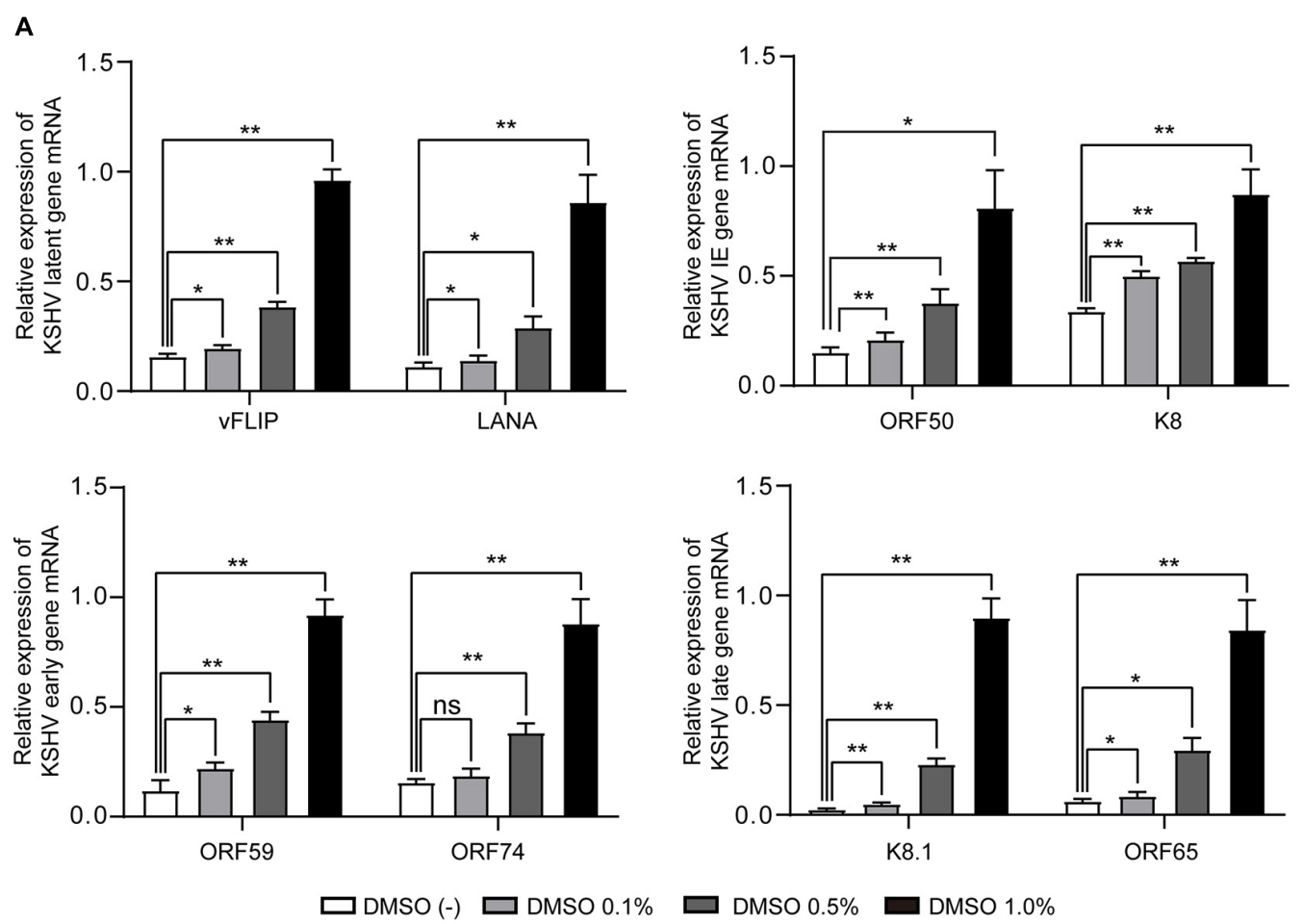

B

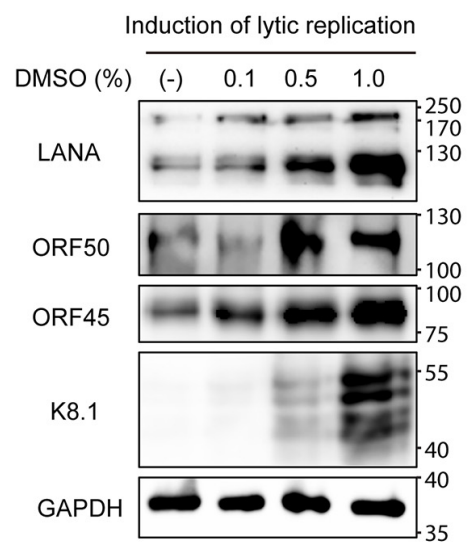

C

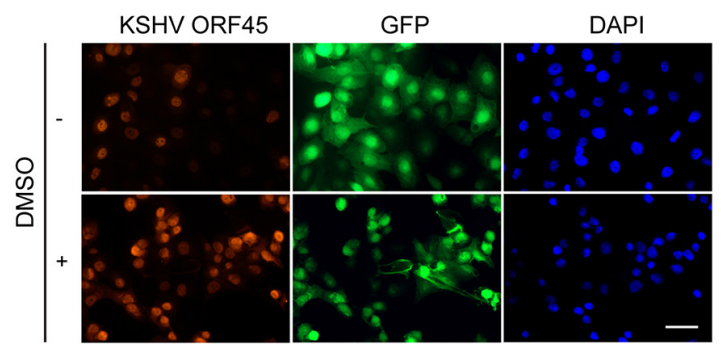

KSHV K8.1

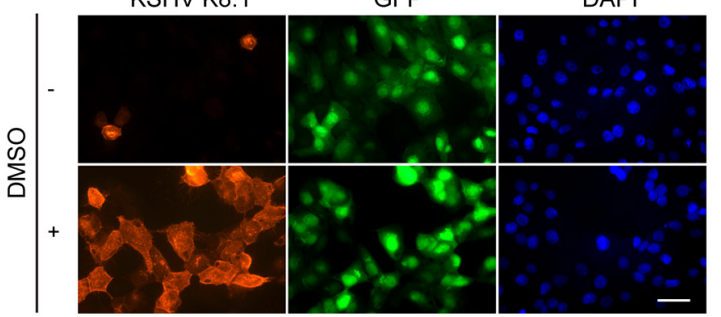

FIGURE 3 | Increased expression of viral genes by DMSO during lytic replication of KSHV. iSLK BAC16 cells were treated with various concentrations of DMSO during lytic replication. After $24 \mathrm{~h}$, total RNA and protein were isolated to analyze viral gene expression. (A) RT-qPCR analysis of mRNA expression of KSHV viral genes. Data are shown as the mean $\pm \mathrm{SD}, n=3,{ }^{*} p<0.05$, and ${ }^{* *} p<0.01$. (B) Western blot analysis of the indicated viral proteins in iSLK BAC16 with induction of Iytic replication. (C) Immunofluorescence assay (IFA) for the indicated viral proteins in iSLK BAC16 with induction of lytic replication. DMSO (1\%) was treated to cells at the beginning of Iytic replication. IFA was analyzed at $24 \mathrm{~h}$ after induction. Scale bar $=50 \mu \mathrm{m}$.

by assessment of GFP expression because this recombinant KSHV contained a GFP cassette. Interestingly, KSHV infectivity in HUVECs was increased for viruses harvested by treatment with DMSO compared with those without DMSO treatment (Figures 2A-C). In flow cytometry analysis, KSHV extracted from iSLK BAC16 cells treated with 1\% DMSO showed an approximately 2.5 -fold increase in infection rate compared with KSHV extracted from untreated cells (Figures 2B,C). These results indicated that KSHV extracted by adding DMSO resulted in a higher infection rate in HUVECs. A previous study reported 
A

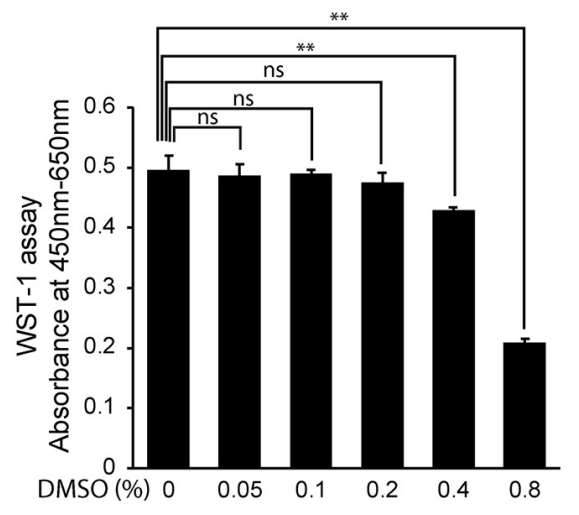

C

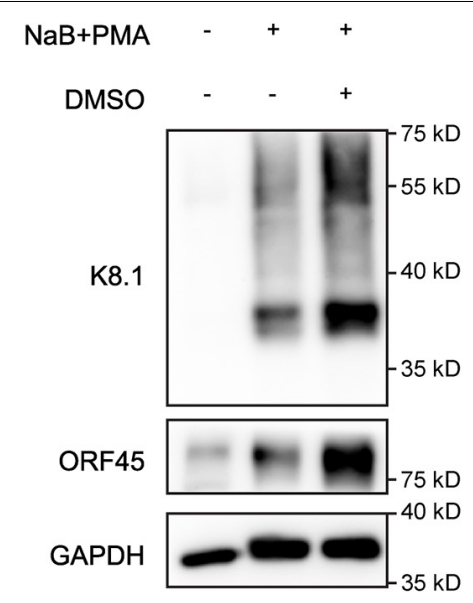

B
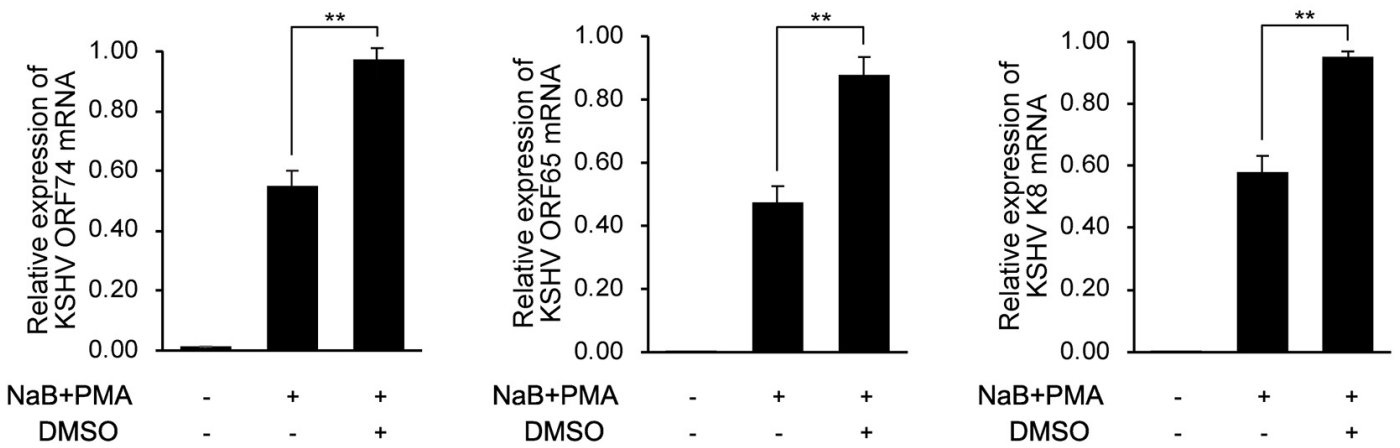

FIGURE 4 | Enhanced expression of KSHV Iytic genes in BCBL-1 cells by DMSO during reactivation. (A) Cell viability with various concentrations of DMSO. After $24 \mathrm{~h}$ of DMSO treatment, cell viabilities were analyzed by WST-1 assay. Data are shown as the mean $\pm \mathrm{SD}, n=3$, ns, not significant; ${ }^{* *} p<0.01$. (B) RT-qPCR analysis of mRNA expression of KSHV viral genes. Reactivation of KSHV was induced in BCBL-1 by treatment with sodium butyrate (NaB, $0.3 \mathrm{mM})$ and phorbol-13-acetate (PMA, $20 \mathrm{ng} / \mathrm{mL})$. DMSO (0.2\%) was treated at the beginning of reactivation. RNA or protein lysate was extracted at $48 \mathrm{~h}$ after induction of lytic replication. Data are shown as the mean $\pm \mathrm{SD}, n=3$, and ${ }^{* *} p<0.01$. (C) Western blot analysis of KSHV ORF45 and K8.1 in BCBL-1 cells.

that DMSO could affect protein structure and interaction (Chan et al., 2017). To investigate whether DMSO enhances infectivity of KSHV by modifying protein structures and receptor-ligand interaction, the isolated KSHV from iSLK BAC16 cells without DMSO treatment was infected with HUVECs together with DMSO (Supplementary Figure 1). Our results did not show any significant change of KSHV infectivity with DMSO treatment, suggesting that DMSO would not affect KSHV infectivity. To investigate whether the increase in KSHV infectivity was correlated with the increase in virion production, the extracted virions from each experimental group were analyzed by western blotting and qPCR. In western blot analysis, KSHV envelope protein K8.1, KSHV tegument protein ORF45, and KSHV capsid protein ORF65 were analyzed using specific antibodies (Figure 2D). The same volume of virus was extracted by adding various concentrations of DMSO, and the expression levels of all analyzed viral proteins were increased in proportion to DMSO. In addition, genomic DNA was extracted from KSHV virions, and KSHV genomic DNA was quantified using qPCR analysis of KSHV ORF26 (Figure 2E). KSHV genomic DNA was also increased by DMSO in a concentration-dependent manner. Our results indicated that the addition of DMSO enhanced KSHV virion production during the induction of lytic replication in KSHV iSLK BAC16 cells.

\section{Dimethyl Sulfoxide Enhanced Kaposi's Sarcoma-Associated Herpesvirus Viral Gene Expression in iSLK BAC16 Cells During Lytic Replication}

Because viral production was increased by additional DMSO during lytic replication, we next evaluated whether DMSOmediated virus production was correlated with lytic replication of KSHV. To this end, after treating iSLK BAC16 cells with various concentrations of DMSO for $24 \mathrm{~h}$ during lytic replication, total RNA, and cell lysates were extracted to analyze KSHV viral gene expression. During lytic replication, all analyzed mRNA expression of KSHV viral genes was significantly increased 


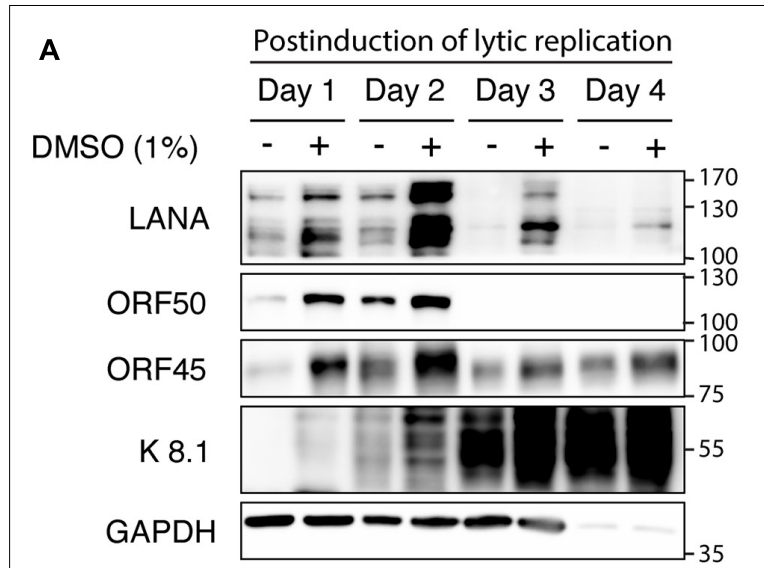

B

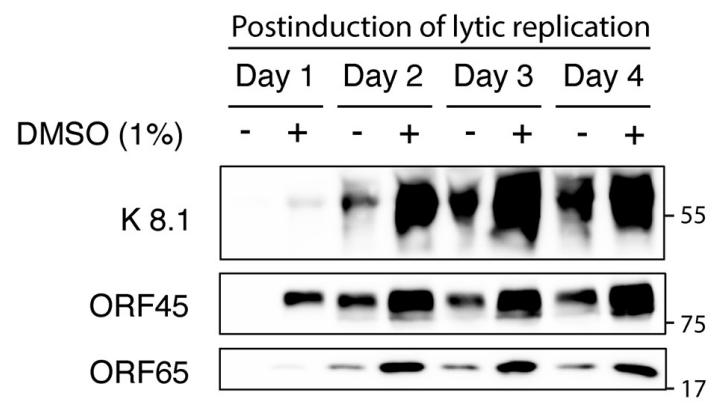

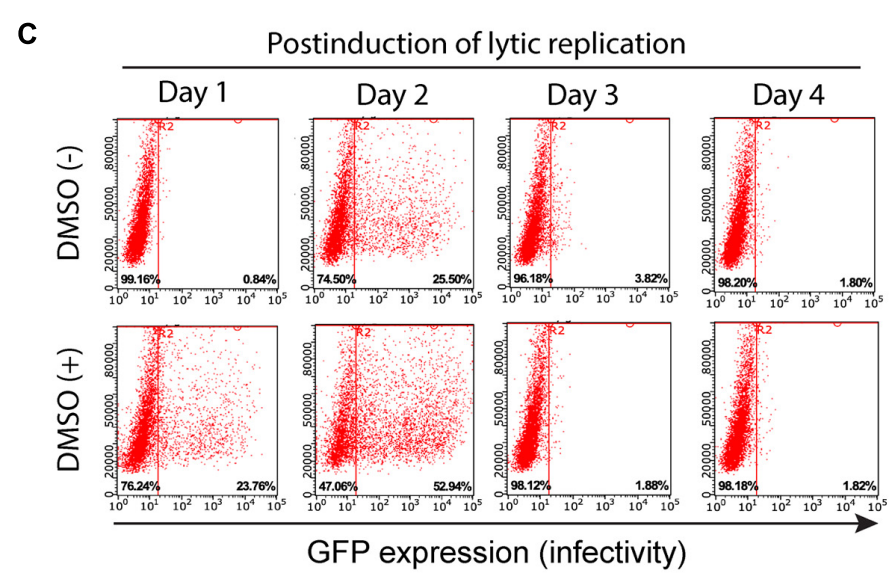

D

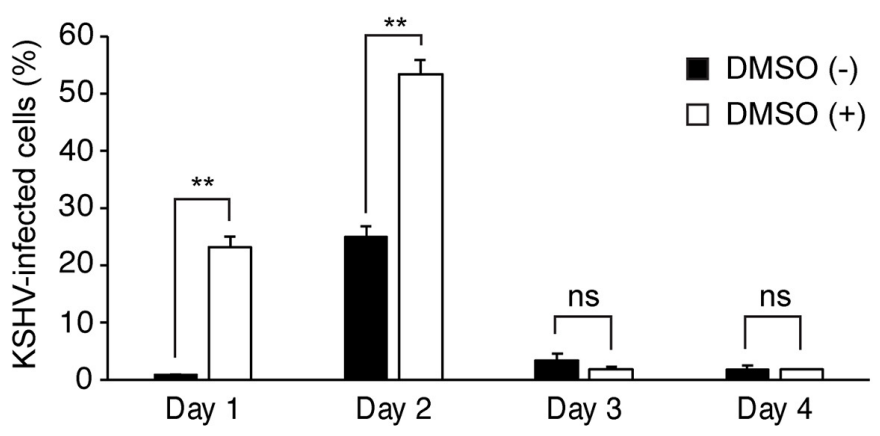

FIGURE $\mathbf{5}$ | Time kinetic analysis of changes in viral gene expression and virus production of KSHV by DMSO treatment during reactivation of KSHV. (A) Western blot analysis for KSHV gene expression in iSLK BAC16 cells with DMSO. iSLK BAC16 cells were treated with DMSO and inducing agents for lytic replication together, then analyzed indicated viral proteins. (B) Western blot analysis of KSHV proteins of the isolated KSHV from iSLK BAC16 cells. After induction of Iytic replication, KSHV was extracted from the supernatants of iSLK BAC16 cells at the indicated time points. The same volume of extracted KSHV from each experimental condition was analyzed. (C,D) The flow cytometric analysis of GFP expression in KSHV-infected HUVECs. The isolated KSHV from each condition infected with HUVECs, followed by analyzing GFP expression by flow cytometry at $24 \mathrm{~h}$ after infection. Representative results of the flow cytometric analysis of GFP expression in KSHV-infected HUVECs (C). Analysis of KSHV infectivity (D). Data are shown as the mean $\pm \mathrm{SD}, n=3$, and ${ }^{* *} p<0.01$. ns, not significant.

by DMSO treatment in a concentration-dependent manner (Figure 3A). Similarly, DMSO increased the expression of viral lytic proteins, such as ORF50, ORF45, and K8.1 in iSLK BAC16 cells under lytic replication (Figure 3B). Interestingly, mRNA and protein expression levels of latent genes, such as vFLIP (ORF71) and LANA (ORF73), were significantly increased by DMSO. Together, these results suggested that DMSO may affect not only lytic genes but also overall KSHV gene expression. To investigate whether DMSO increased the number of reactivating cells or increased copies of lytic gene transcripts within a reactivating cell, the expressions of KSHV lytic genes including ORF45 and K8.1 were analyzed by IFA (Figure 3C). The number of cells expressing ORF 45 and K8.1 was increased by DMSO treatment compared to the control group. These results indicated that DMSO could induce lytic replication in more cells. However, the possibility that DMSO increases gene expression in single cells cannot be completely excluded. To investigate DMSO also affects lytic replication of primary effusion lymphoma (PEL) cell line with KSHV, BCBL1 was treated with a non-toxic concentration of DMSO $(0.2 \%)$ during induction of lytic replication (Figure 4A). The lytic genes of KSHV were also enhanced by DMSO in BCBL-1 cells (Figures $4 \mathrm{~B}, \mathrm{C}$ ), suggesting DMSO would have a similar stimulating effect on lytic replication on iSLK BAC16 cells and BCBL-1 cells.

\section{Dimethyl Sulfoxide Induced Faster Lytic Reactivation of Kaposi's Sarcoma-Associated Herpesvirus in iSLK BAC16 Cells}

Next, we analyzed time-kinetics for viral gene expression, virion production, and infectivity of produced KSHV to determine whether increased viral production with DMSO indeed increased viral production or simply rapidly induced reactivation of KSHV. In iSLK BAC16 cells with induction of lytic replication, we found that all analyzed viral proteins were increased in DMSO-treated cells than in no treated control (Figure 5A). Because we optimized our induction conditions for maximal virus production in a short time, most cells were dead after 2 days of induction. While the previous studies used doxycycline at $1 \mu \mathrm{g} / \mathrm{mL}$ and sodium butyrate at $1 \mathrm{mM}$ (Brulois et al., 2012), we 

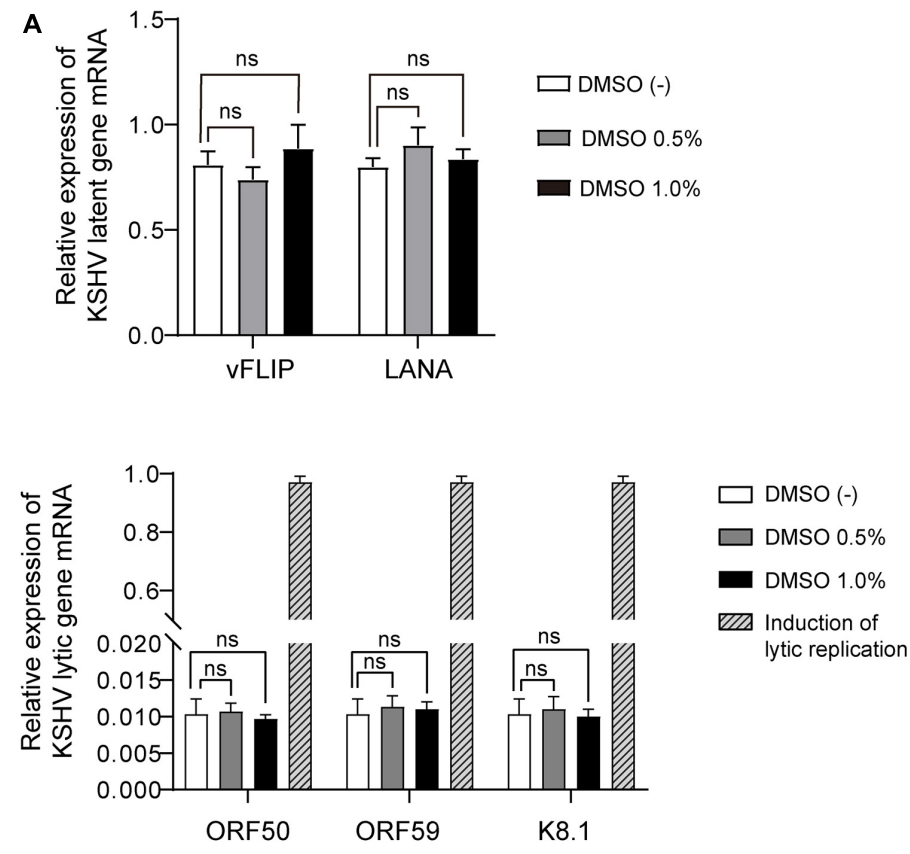

B

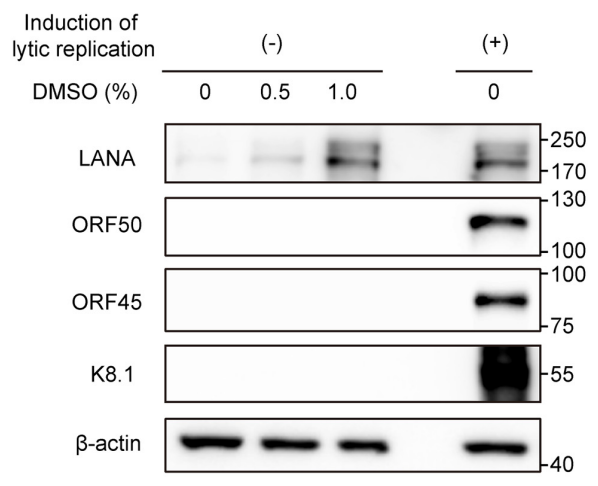

FIGURE 6 | Differential expression of viral genes induced by DMSO during latent KSHV infection. iSLK BAC16 cells were treated with various concentrations of DMSO for $24 \mathrm{~h}$. Next, total RNA and protein were isolated to analyze viral gene expression. (A) RT-qPCR analysis of mRNA expression of KSHV viral genes. Data are shown as the mean $\pm \mathrm{SD}, n=3$, and ns, not significant. (B) Western blot analysis of the indicated viral proteins in iSLK BAC16 with (+) or without induction of lytic replication (-).

used doxycycline at $50 \mu \mathrm{g} / \mathrm{mL}$ and sodium butyrate at $1.2 \mathrm{mM}$ for induction of lytic replication. Therefore, after 3 days of induction, most analyzed proteins except for the late protein K8.1 were decreased compared at 2 days of induction. In the extracted $\mathrm{KSHV}$ from the supernatant, all viral proteins from the DMSO-treated cells were also increased at all analyzed time points (Figure 5B). We found that the virus was produced from the cells treated with DMSO 1 day after induction of lytic replication, and the quantitative difference was also maintained until the fourth day. Therefore, it was confirmed that DMSO has the effect of not only enhancing KSHV production, but also induction of faster lytic replication and viral production (Figures 5A,B). To determine the infectivity of $\mathrm{KSHV}$ is proportional to the viral proteins of each extracted KSHV, the isolated KSHV was infected with HUVECs (Figures 5C,D). In the infected cells with virus extracted after induction of lytic replication for 1 day, GFP expression was not observed in the virus from the control cells not treated with DMSO. However, the KSHV from DMSO-treated cells showed high infectivity in HUVECs, which is consistent with the amount of virus protein analyzed in Figure 5B. At 2 days of induction, it was confirmed that the infection of the virus extracted from DMSO-treated cells was increased by about two times compared to the control. Regardless of DMSO treatment, the infectivity of the isolated KSHV was not observed after 3 days of induction of lytic replication. Since, in our experimental condition, most cells die after 2 days of induction, the virion may be inactivated in an acidic condition of culture media with dead cells.

\section{Dimethyl Sulfoxide Alone Did Not Induce Lytic Replication of Kaposi's Sarcoma-Associated Herpesvirus in iSLK BAC16 Cells}

To explore the effects of DMSO on KSHV gene expression in latent infection, total RNA and cell lysates were extracted after treating iSLK BAC16 cells with various concentrations of DMSO for $24 \mathrm{~h}$. During latent infection, we did not find any evidence that DMSO alone induced lytic replication without inducing reagents (Figure 6). mRNA expressions for latent and lytic genes were not affected by DMSO (Figure 6A). In protein levels, KSHV ORF50, ORF45, and K8.1 were not detected in DMSO treated iSLK BAC16 cells without induction of lytic replication (Figure 6B).

\section{Dimethyl Sulfoxide Enhanced the Lytic Replication Cycle by Induction of C-Jun $\mathrm{N}$-Terminal Kinase Phosphorylation}

Some cell signaling pathways, including the MAPK and phosphatidylinositol 3-kinase/AKT pathways, are known to be associated with KSHV lytic replication (Ford et al., 2006; Pan et al., 2006; Xie et al., 2008; Peng et al., 2010). Therefore, we next performed western blot analysis of several phosphorylated signaling proteins to investigate whether known signaling pathways related to KSHV lytic replication were associated with DMSO treatment in iSLK BAC16 cells under lytic replication. Although the levels of phospho-AKT, phosphoERK, and phospho-p38 MAPK were not increased significantly 
A

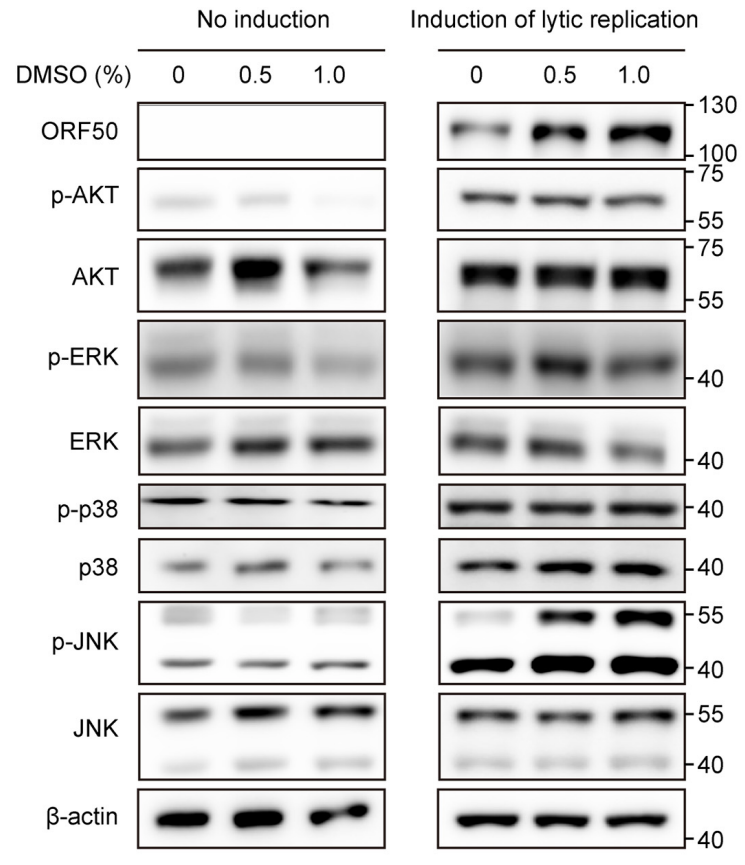

B

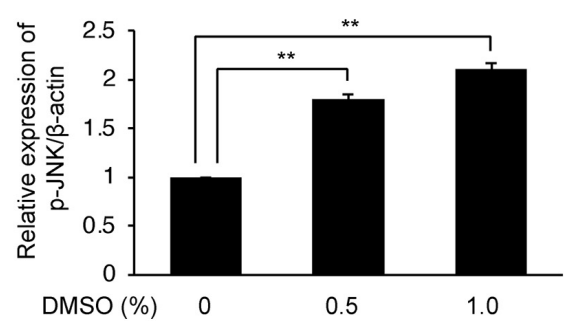

C

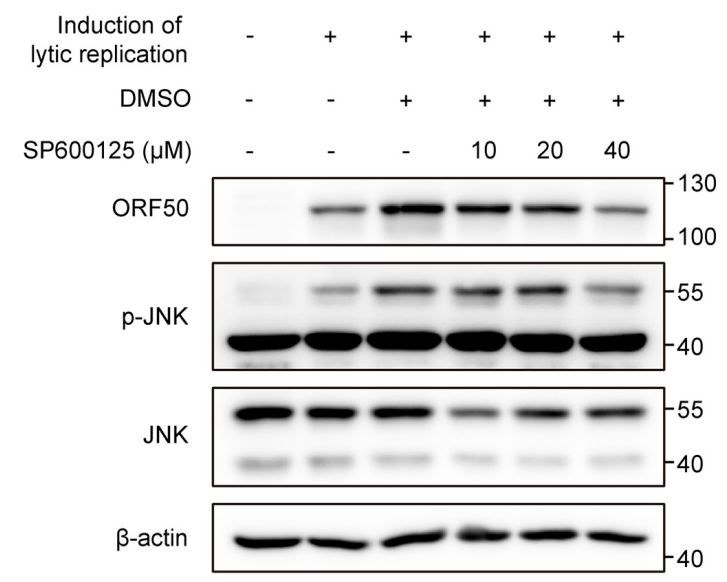

D

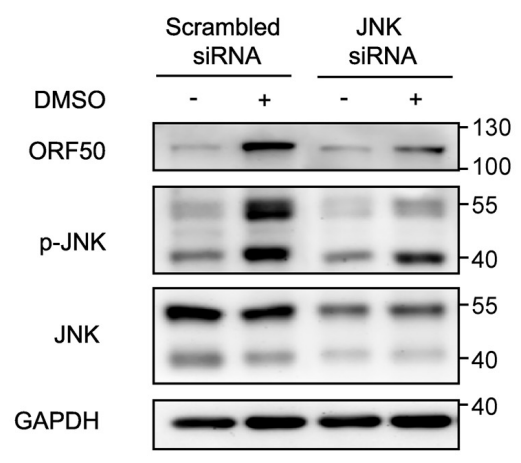

FIGURE 7 | Enhanced lytic replication by DMSO was mediated by increased phosphorylation of JNK. Cells were then treated with DMSO during lytic replication. No induction samples were treated with DMSO alone. Lytic replication was induced in iSLK BAC16 cells with doxycycline and sodium butyrate. (A) Western blot analysis of signaling molecules related to lytic replication of KSHV. (B) Densitometric analysis of phospho-JNK/ $\beta$-actin levels in iSLK BAC16 cells treated with DMSO and lytic replication-inducing agents. Data are shown as the mean \pm SD, $n=3$, and ${ }^{* *} p<0.01$. (C,D) Effects of the JNK inhibitor SP600125 or siRNA-mediated suppression of JNK on KSHV Iytic replication in DMSO-treated iSLK BAC16 cells. Cells were treated with 10, 20, or $40 \mu \mathrm{M}$ of SP600125 at the beginning of lytic activation with DMSO. After $24 \mathrm{~h}$, cell lysates were collected, and the indicated proteins were analyzed by western blot analysis with specific antibodies (C). For suppression of JNK with siRNA, iSLK BAC16 cells were transfected with JNK-specific small interfering RNA (JNK siRNA) and scrambled sequence control siRNA (scrambled siRNA) $24 \mathrm{~h}$ before lytic activation (D).

by DMSO, phospho-JNK levels were increased in DMSOtreated iSLK BAC16 cells compared with that in untreated control cells (Figures 7A,B). In BCBL-1 cells, phospho-JNK was also increased by DMSO treatment during induction of lytic replication (Supplementary Figure 2). The JNKspecific inhibitor SP600125 was used to determine whether the enhancement of lytic replication in DMSO-treated cells was mediated by increased phosphorylation of JNK. Notably, at non-cell killing concentrations, SP600125 suppressed not only phospho-JNK levels but also KSHV ORF50 expression (Figure 7C and Supplementary Figure 3). To confirm the association of JNK and enhanced lytic replication by DMSO, siRNA for JNK was applied to iSLK BAC16 cells during lytic replication (Figure 7D). Suppression of JNK by siRNA attenuated ORF50 expression enhanced by DMSO treatment during lytic replication, which is consistent with that by the inhibition of JNK using SP600125 shown in Figure 7C. Taken together, our results suggested that JNK phosphorylation was associated with increased lytic replication induced by DMSO.

\section{Suppression of Dimethyl Sulfoxide-Mediated Enhancement of Kaposi's Sarcoma-Associated Herpesvirus Production by Inhibition of C-Jun N-Terminal Kinase Pathway}

Because JNK inhibition suppressed lytic replication of KSHV in DMSO-treated iSLK BAC16 cells (Figure 7C), we investigated whether JNK inhibitor, SP600125, also suppressed DMSOmediated enhancement of KSHV production. After isolation 


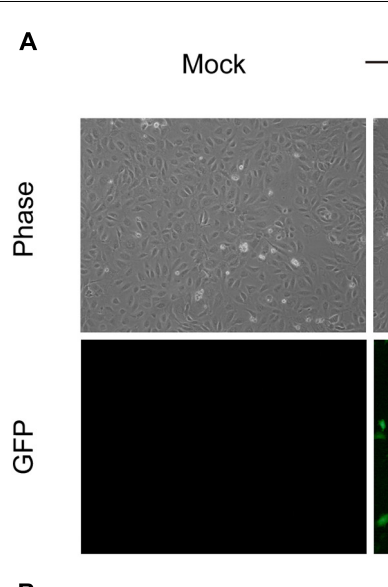

B

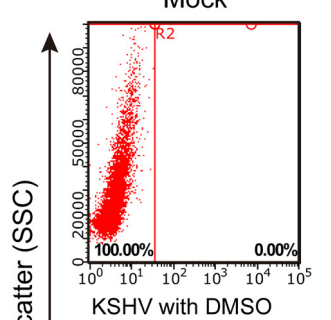

$\mathrm{KSHV}$ with DMSO

믐

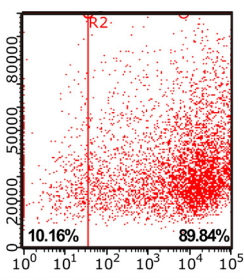

KSHV-infected cells (GFP)

D

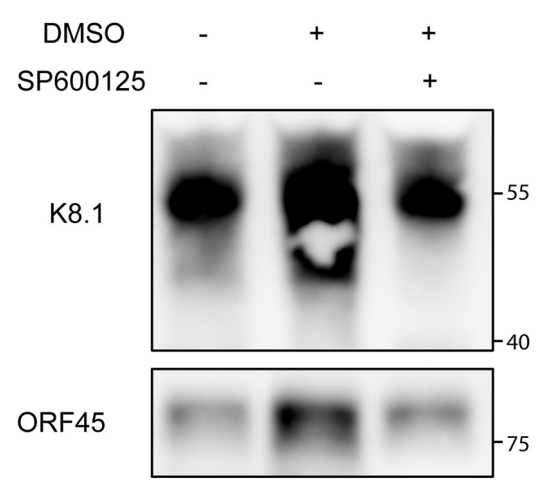

KSHV produced with
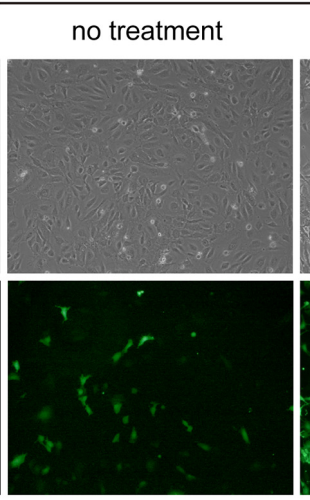

KSHV with DMSO (-)

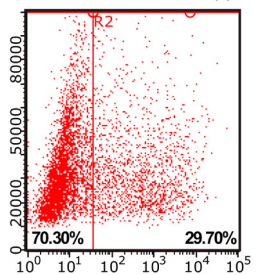

KSHV with DMSO + SP600125

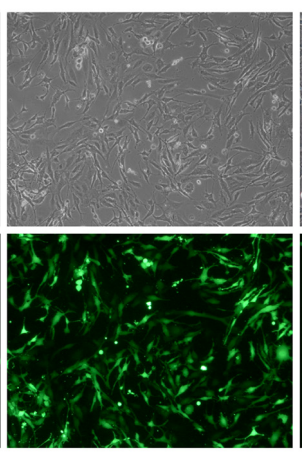

C

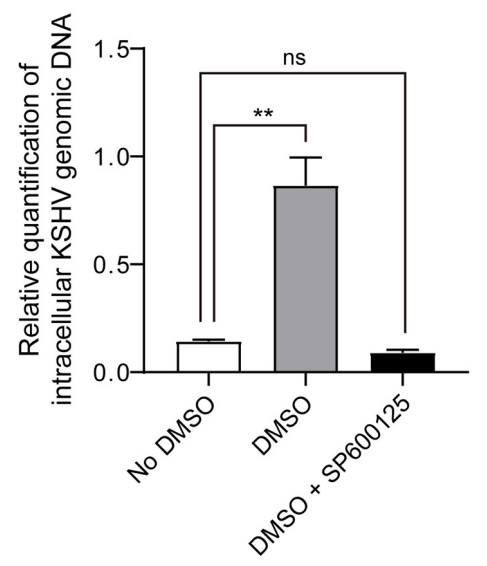

E

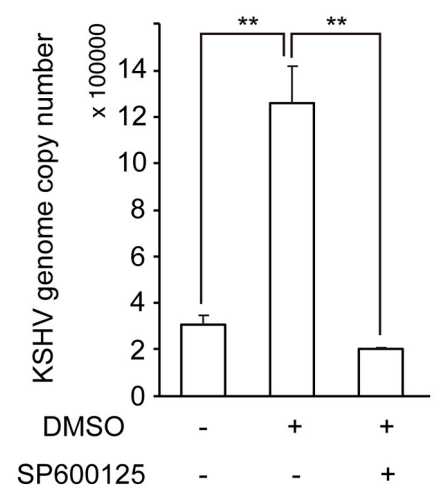

FIGURE 8 | JNK inhibitor suppressed DMSO-mediated enhancement of KSHV virion production in iSLK BAC16 cells. iSLK BAC16 cells were treated with the JNK inhibitor SP600125 (20 $\mu \mathrm{M})$ at the beginning of lytic activation with DMSO. After 2 days, KSHV was harvested from each culture supernatant. The same volume of KSHV from each group was used to infect HUVECs. (A) GFP expression of KSHV-infected cells, as evaluated by fluorescence microscopy. After 24 h of infection, GFP expression was observed using fluorescence microscopy. Scale bar $=250 \mu \mathrm{m}$. (B) Representative results of flow cytometric analysis of GFP expression in KSHV-infected HUVECs. GFP-expressing cells from panel (A) were detached and analyzed by flow cytometry. $X$ - and $Y$-axis indicate GFP expression and side scatter, respectively. The numbers in the right lower corners of each panel indicate the percentage of GFP-positive cells. (C) qPCR for KSHV genomic DNA in KSHV-infected HUVECs. KSHV from each experimental group (no DMSO, DMSO alone, and DMSO with SP600125) was used to infect HUVECs, and genomic DNA was extracted at $4 \mathrm{~h}$ postinfection. Data are shown as the mean $\pm \mathrm{SD}, n=3$, and ns, not significant. ${ }^{\star *} p<0.01$. (D,E) Attenuated KSHV production by JNK inhibitor. After induction of lytic replication, KSHV was extracted from the supernatants of iSLK BAC16 cells. iSLK BAC16 cells were treated with DMSO or SP600125 during lytic replication. The same volume of extracted KSHV from each experimental condition was analyzed. Western blot analysis of KSHV proteins of the isolated KSHV virions (D). Analysis of KSHV genome copy numbers from the produced virions (E). Genomic DNA was isolated from the extracted virions and analyzed by qPCR with specific primers targeting KSHV ORF26. Data are shown as the mean $\pm \mathrm{SD}, n=3$, and ${ }^{* *} p<0.01$. 
of KSHV from iSLK BAC16 cells, HUVECs were infected with the virus. As shown in Figures 8A,B, GFP-expressing cells were prominent in DMSO-treated KSHV-infected cells. KSHV produced from iSLK BAC16 cells treated with both DMSO and SP600125 showed less infectivity than KSHV produced with DMSO alone (Figure 8B and Supplementary Figure 4), suggesting that the JNK inhibitor suppressed the DMSO-mediated enhancement of KSHV production in iSLK BAC16 cells. KSHV genomic DNA in KSHV-infected HUVECs was significantly suppressed following infection of cells with KSHV extracted from DMSO- and SP600125-treated iSLK BAC16 cells compared with that in cells treated with DMSO alone (Figure $\mathbf{8 C}$ ), consistent with the results shown in Figures 8A,B. As a result of analyzing $\mathrm{KSHV}$ isolated from the supernatant under each condition, the virus proteins and KSHV genome copy number were suppressed in the SP600125-treated group (Figures 8D,E) and siRNA for JNK-transfected group (Supplementary Figure 5), suggesting that DMSO-mediated enhancement of KSHV production would be associated with the JNK pathway.

\section{DISCUSSION}

We found that DMSO enhanced KSHV lytic gene expression, resulting in at least a twofold increase in KSHV virion production. During lytic replication, DMSO increased both viral mRNA and protein expression. Although KSHV LANA protein was upregulated by DMSO, even during latent infection, DMSO alone did not induce lytic replication under our experimental conditions. We also demonstrated that the phosphorylation of JNK was associated with enhanced lytic replication and KSHV production induced by DMSO.

Although DMSO is the most commonly used solvent for cryopreservation and in vitro assays, its biological effects are frequently overlooked. A recent study showed that DMSO significantly affects not only the expression of mRNA/microRNA but also DNA methylation in three-dimensional cardiac and hepatic microtissues (Verheijen et al., 2019). Several studies have reported that DMSO affects the life cycles of viruses. For example, the replication or virus of fowl plague virus (Scholtissek and Muller, 1988), human immunodeficiency virus 1 (Taddeo et al., 2000), and duck hepatitis B virus (Tsuei et al., 1979) is increased by DMSO. In addition, DMSO induces reactivation of murine cytomegalovirus in latently infected mouse spleens (Blackett et al., 1987).

In many previous studies on KSHV, DMSO has been frequently used in vitro studies as a vehicle for chemical agents (Lee et al., 2014; Jeon et al., 2017; Kang et al., 2021). Intriguingly, we found that DMSO enhanced the expression of KSHV viral proteins, including LANA, ORF50, ORF45, and K8.1, during lytic replication. The phosphorylation of JNK was found to be associated with DMSO-mediated enhancement of lytic replication of KSHV because JNK phosphorylation was increased as ORF50 expression was increased by DMSO, whereas inhibition of JNK phosphorylation suppressed both lytic replication and KSHV production. A previous study demonstrated that DMSO induced JNK phosphorylation in human endothelial cells (Liang et al., 2011). Indeed, DMSO is known to have diverse biological effects on cells. Therefore, we could not exclude the possibility that another mechanism may be associated with the enhanced lytic replication of KSHV by DMSO.

In latent infection, the expression of KSHV LANA protein was also increased by treatment with DMSO. However, unlike the results in lytic replication, wherein both LANA RNA and protein were upregulated by DMSO, we did not observe increased mRNA levels during latent infection. These results revealed that DMSO affected viral gene expression during both latent and lytic replication of KSHV; however, the underlying mechanisms may be different in latent and lytic replication. Since DMSO did not increase transcript levels for latent genes in the same condition, DMSO may regulate LANA expression in the translation or protein degradation process. Further studies are required to evaluate the exact mechanisms through which DMSO regulates LANA expression in latent KSHV infection.

In summary, this is the first study showing that DMSO enhanced lytic replication of $\mathrm{KSHV}$ and virion production during induced lytic replication. We showed that DMSO effectively increased KSHV production in the KSHV-producing cell line iSLK BAC16. Our findings may be helpful for studies requiring larger amounts of KSHV virions.

\section{DATA AVAILABILITY STATEMENT}

The original contributions presented in the study are included in the article/Supplementary Material, further inquiries can be directed to the corresponding author.

\section{AUTHOR CONTRIBUTIONS}

S-KK and M-SL designed the study. S-KK, M-JL, JL, and H-HR performed the experiments and analyzed the data. S-KK, H-HR, and M-SL wrote the manuscript. All authors read and approved the final manuscript.

\section{FUNDING}

This research was supported by the Mid-Career Research Program through the National Research Foundation of Korea (NRF), project number NRF-2019R1A2C2083947 to M-SL.

\section{ACKNOWLEDGMENTS}

We thank the members of Lee's laboratory for their technical assistance and helpful discussions.

\section{SUPPLEMENTARY MATERIAL}

The Supplementary Material for this article can be found online at: https://www.frontiersin.org/articles/10.3389/fmicb.2021. 778525/full\#supplementary-material 


\section{REFERENCES}

Aguilar, J. S., Roy, D., Ghazal, P., and Wagner, E. K. (2002). Dimethyl sulfoxide blocks herpes simplex virus-1 productive infection in vitro acting at different stages with positive cooperativity. Application of micro-array analysis. BMC Infect. Dis. 2:9. doi: 10.1186/1471-2334-2-9

Blackett, S., Gupta, A., and Mims, C. A. (1987). DMSO induces reactivation of cytomegalovirus in vitro from spleens of latently infected mice. Brief report. Arch. Virol. 93, 273-278. doi: 10.1007/BF01310980

Boshoff, C., and Weiss, R. (2002). AIDS-related malignancies. Nat. Rev. Cancer 2, 373-382. doi: 10.1038/nrc797

Brayton, C. F. (1986). Dimethyl sulfoxide (DMSO): a review. Cornell Vet. 76, 61-90.

Brulois, K. F., Chang, H., Lee, A. S., Ensser, A., Wong, L. Y., Toth, Z., et al. (2012). Construction and manipulation of a new Kaposi's sarcoma-associated herpesvirus bacterial artificial chromosome clone. J. Virol. 86, 9708-9720. doi: 10.1128/JVI.01019- 12

Cesarman, E., Chang, Y., Moore, P. S., Said, J. W., and Knowles, D. M. (1995). Kaposi's sarcoma-associated herpesvirus-like DNA sequences in AIDS-related body-cavity-based lymphomas. N. Engl. J. Med. 332, 1186-1191. doi: 10.1056/ NEJM199505043321802

Chan, D. S., Kavanagh, M. E., McLean, K. J., Munro, A. W., Matak-Vinkovic, D., Coyne, A. G., et al. (2017). Effect of DMSO on protein structure and interactions assessed by collision-induced dissociation and unfolding. Anal. Chem. 89, 9976-9983. doi: 10.1021/acs.analchem.7b02329

Chang, J., Renne, R., Dittmer, D., and Ganem, D. (2000). Inflammatory cytokines and the reactivation of Kaposi's sarcoma-associated herpesvirus lytic replication. Virology 266, 17-25. doi: 10.1006/viro.199 9.0077

Chang, M., Brown, H. J., Collado-Hidalgo, A., Arevalo, J. M., Galic, Z., Symensma, T. L., et al. (2005). beta-Adrenoreceptors reactivate Kaposi's sarcoma-associated herpesvirus lytic replication via PKA-dependent control of viral RTA. J. Virol. 79, 13538-13547. doi: 10.1128/JVI.79.21.13538-13547.2005

Chang, Y., Cesarman, E., Pessin, M. S., Lee, F., Culpepper, J., Knowles, D. M., et al. (1994). Identification of herpesvirus-like DNA sequences in AIDSassociated Kaposi's sarcoma. Science 266, 1865-1869. doi: 10.1126/science.79 97879

Cohen, A., Brodie, C., and Sarid, R. (2006). An essential role of ERK signalling in TPA-induced reactivation of Kaposi's sarcoma-associated herpesvirus. J. Gen. Virol. 87(Pt 4), 795-802. doi: 10.1099/vir.0.81619-0

Ford, P. W., Bryan, B. A., Dyson, O. F., Weidner, D. A., Chintalgattu, V., and Akula, S. M. (2006). Raf/MEK/ERK signalling triggers reactivation of Kaposi's sarcoma-associated herpesvirus latency. J. Gen. Virol. 87(Pt 5), 1139-1144. doi: 10.1099/vir.0.81628-0

Jeon, H., Yoo, S. M., Choi, H. S., Mun, J. Y., Kang, H. G., Lee, J., et al. (2017). Extracellular vesicles from KSHV-infected endothelial cells activate the complement system. Oncotarget 8, 99841-99860. doi: 10.18632/oncotarget. 21668

Kang, S. K., Kang, Y. H., Yoo, S. M., Park, C., Kim, H. S., and Lee, M. S. (2021). HMGB1 knockout decreases Kaposi's sarcoma-associated herpesvirus virion production in iSLK BAC16 cells by attenuating viral gene expression. J. Virol. 95:e0079921. doi: 10.1128/jvi.00799-21

Lee, M. S., Jones, T., Song, D. Y., Jang, J. H., Jung, J. U., and Gao, S. J. (2014). Exploitation of the complement system by oncogenic Kaposi's sarcomaassociated herpesvirus for cell survival and persistent infection. PLoS Pathog. 10:e1004412. doi: 10.1371/journal.ppat.1004412

Liang, C., Xue, Z., Cang, J., Wang, H., and Li, P. (2011). Dimethyl sulfoxide induces heme oxygenase-1 expression via JNKs and Nrf2 pathways in human umbilical vein endothelial cells. Mol. Cell Biochem. 355, 109-115. doi: 10.1007/s11010011-0844-Z

Lu, F., Zhou, J., Wiedmer, A., Madden, K., Yuan, Y., and Lieberman, P. M. (2003). Chromatin remodeling of the Kaposi's sarcoma-associated herpesvirus ORF50 promoter correlates with reactivation from latency. J. Virol. 77, 11425-11435. doi: 10.1128/jvi.77.21.11425-11435.2003

Pan, H., Xie, J., Ye, F., and Gao, S. J. (2006). Modulation of Kaposi's sarcomaassociated herpesvirus infection and replication by MEK/ERK, JNK, and p38 multiple mitogen-activated protein kinase pathways during primary infection. J. Virol. 80, 5371-5382. doi: 10.1128/JVI.02299-05
Peng, L., Wu, T. T., Tchieu, J. H., Feng, J., Brown, H. J., Feng, J., et al. (2010). Inhibition of the phosphatidylinositol 3-kinase-Akt pathway enhances gamma2 herpesvirus lytic replication and facilitates reactivation from latency. J. Gen. Virol. 91(Pt 2), 463-469. doi: 10.1099/vir.0.015073-0

Polizzotto, M. N., Uldrick, T. S., Wyvill, K. M., Aleman, K., Marshall, V., Wang, V., et al. (2016). Clinical features and outcomes of patients with symptomatic kaposi sarcoma herpesvirus (KSHV)-associated inflammation: prospective characterization of KSHV inflammatory cytokine syndrome (KICS). Clin. Infect. Dis. 62, 730-738. doi: 10.1093/cid/civ996

Powles, T., Stebbing, J., Bazeos, A., Hatzimichael, E., Mandalia, S., Nelson, M., et al. (2009). The role of immune suppression and HHV-8 in the increasing incidence of HIV-associated multicentric Castleman's disease. Ann. Oncol. 20, 775-779. doi: 10.1093/annonc/mdn697

Scholtissek, C., and Muller, K. (1988). Effect of dimethylsulfoxide (DMSO) on virus replication and maturation. Arch. Virol. 100, 27-35. doi: 10.1007/BF01310905

Shin, H. J., DeCotiis, J., Giron, M., Palmeri, D., and Lukac, D. M. (2014). Histone deacetylase classes I and II regulate Kaposi's sarcoma-associated herpesvirus reactivation. J. Virol. 88, 1281-1292. doi: 10.1128/JVI.02665-13

Taddeo, B., Nickoloff, B. J., and Foreman, K. E. (2000). Caspase inhibitor blocks human immunodeficiency virus 1-induced T-cell death without enhancement of HIV-1 replication and dimethyl sulfoxide increases HIV-1 replication without influencing T-cell survival. Arch. Pathol. Lab. Med. 124, 240-245. doi: 10.5858/2000-124-0240-CIBHIV

Tanaka, J., Kamiya, S., Ogura, T., Sato, H., Ogura, H., and Hatano, M. (1985). Effect of dimethyl sulfoxide on interaction of human cytomegalovirus with host cell: conversion of a nonproductive state of cell to a productive state for virus replication. Virology 146, 165-176. doi: 10.1016/0042-6822(85)90001-7

Tsuei, D., Haubenstock, H., Revoltella, R., and Friend, C. (1979). Virus production and hemoglobin synthesis in variant lines of dimethyl sulfoxide-treated Friend erythroleukemia cells. J. Virol. 31, 178-183. doi: 10.1128/JVI.31.1.178-183.1979

Verheijen, M., Lienhard, M., Schrooders, Y., Clayton, O., Nudischer, R., Boerno, S., et al. (2019). DMSO induces drastic changes in human cellular processes and epigenetic landscape in vitro. Sci. Rep. 9:4641. doi: 10.1038/s41598-019-4 0660-0

West, P. G., Aldrich, B., Hartwig, R., and Haller, G. J. (1988). Enhanced detection of cytomegalovirus in confluent MRC-5 cells treated with dexamethasone and dimethyl sulfoxide. J. Clin. Microbiol. 26, 2510-2514. doi: 10.1128/jcm.26.12. 2510-2514.1988

Xie, J., Ajibade, A. O., Ye, F., Kuhne, K., and Gao, S. J. (2008). Reactivation of Kaposi's sarcoma-associated herpesvirus from latency requires MEK/ERK, JNK and p38 multiple mitogen-activated protein kinase pathways. Virology 371, 139-154. doi: 10.1016/j.virol.2007.09.040

Yan, L., Majerciak, V., Zheng, Z. M., and Lan, K. (2019). Towards better understanding of KSHV life cycle: from transcription and posttranscriptional regulations to pathogenesis. Virol. Sin. 34, 135-161. doi: 10.1007/s12250-01900114-3

Yoo, S. M., Zhou, F. C., Ye, F. C., Pan, H. Y., and Gao, S. J. (2005). Early and sustained expression of latent and host modulating genes in coordinated transcriptional program of KSHV productive primary infection of human primary endothelial cells. Virology 343, 47-64. doi: 10.1016/j.virol.2005.08.018

Conflict of Interest: The authors declare that the research was conducted in the absence of any commercial or financial relationships that could be construed as a potential conflict of interest.

Publisher's Note: All claims expressed in this article are solely those of the authors and do not necessarily represent those of their affiliated organizations, or those of the publisher, the editors and the reviewers. Any product that may be evaluated in this article, or claim that may be made by its manufacturer, is not guaranteed or endorsed by the publisher.

Copyright (C) 2021 Kang, Lee, Ryu, Lee and Lee. This is an open-access article distributed under the terms of the Creative Commons Attribution License (CC BY). The use, distribution or reproduction in other forums is permitted, provided the original author(s) and the copyright owner(s) are credited and that the original publication in this journal is cited, in accordance with accepted academic practice. No use, distribution or reproduction is permitted which does not comply with these terms. 